\title{
High-efficiency organic polymer light-emitting heterostructure devices on flexible plastic substrates
}

\author{
Yi He ${ }^{a)}$ and Jerzy Kanicki ${ }^{b}$ \\ Center for Integrated MicroSystems, Department of Electrical Engineering and Computer Science, \\ University of Michigan, Ann Arbor, Michigan 48109-2122
}

(Received 8 July 1999; accepted for publication 8 December 1999)

\begin{abstract}
In this letter, we describe a high-efficiency organic polymer light-emitting heterostructure device with aluminum cathode fabricated on a thin, flexible plastic substrate. The device consists of a hole transporting (amine-fluorene) and an emissive (benzothiadiazole-fluorene) conjugated organic polymer layers. The best heterostructure device has a green light emission and a maximum luminance higher than $2000 \mathrm{~cd} / \mathrm{m}^{2}$. Device shows a maximum emission of $\sim 56.2 \mathrm{~cd} / \mathrm{A}$ and, accordingly, a maximum luminous and external quantum efficiency of $\sim 9.0 \mathrm{~lm} / \mathrm{W}$ and $\sim 15 \%$, respectively. This organic light-emitting diode performance is acceptable for flat panel display applications. (C) 2000 American Institute of Physics. [S0003-6951(00)01206-7]
\end{abstract}

Flexible plastic substrate has a distinct advantage over glass substrate in many applications. Unlike glass, the plastic is usually more robust and compact, has lighter weight, and is more cost effective. The use of the plastic substrates will also enable new product concepts such as curved displays, smart cards, and all plastic electronics. Because of these advantages, in the last decade, plastic substrates have been used in supertwisted nematic liquid-crystal displays $(\mathrm{STN}-\mathrm{LCDs}),{ }^{1} \quad$ active-matrix $\quad$ liquid-crystal displays (AMLCDs), ${ }^{2}$ and organic light-emitting devices (OLEDs). ${ }^{3,4}$ While it is a very promising new technology, the plastic substrate, however, is limited by its low processing temperature, high heat-induced shrinkage, high gas permeability, low chemical resistance, average water and solvent durability, and average transparency. The low thermal durability of the plastic substrate prevents, for example, the high temperature $\left(\geqslant 200^{\circ} \mathrm{C}\right)$ postdeposition thermal annealing of the transparent conducting oxide (TCO), which is typically used in the organic light-emitting device as the transparent electrode. As a result, the TCO on the plastic substrate usually has a lower transparency, a lower conductivity, and a poorer adhesion in comparison with its counterpart on the glass substrate. Another disadvantage of the plastic substrate is its relatively high permeability of the moisture and oxygen that can affect the OLED electrical stability. ${ }^{5}$

In this letter we describe the optoelectrical characteristics of a high-efficiency, bilayer organic polymer lightemitting device with aluminum cathode fabricated on the ITO coated thin, flexible plastic substrate.

The bilayer OLED structure, shown in Fig. 1, consists of a hole transporting $[(\mathrm{HTL}), \sim 170 \AA]$ and an emissive [EL, $\sim 2000 \AA$ ] conducting polymer layers sequentially deposited on the plastic substrate by the spin-coating technique. The polymer absorption and photoluminescence spectra have been published elsewhere. ${ }^{6}$ The schematic energy band diagram of the OLED structure and the cross-section of the flexible plastic substrate are shown in Fig. 1. The lowest unoccupied molecular orbit (LUMO) and the highest occupied molecular orbit (HOMO) levels for both polymers are derived from their cyclic voltammograms and absorption spectra. ${ }^{7}$ The polymer optical band gaps were determined from the onset of the absorption peak that corresponds to the $\pi-\pi^{*}$ transitions of $\pi$ electrons. The polymer Fermi level positions $\left(E_{F}=E_{A}+\right.$ HOMO) were derived from their dark conductivity $(\sigma)$ activation energies $\left(E_{A}\right)$ calculated from the Arrehius plots $\left[\sigma=\sigma_{0} \exp \left(-E_{A} / k T\right)\right]$. The properties of the plastic substrate were reported elsewhere. ${ }^{8}$ Aluminum top cathode electrode was evaporated in a vacuum chamber at the base pressure $\sim 10^{-6}$ Torr through a shadow mask. The OLED electroluminescence (EL) spectra were measured with a charge-coupled device (CCD) spectral analyzer through optical fibers. The CCD spectral analyzer has been calibrated with a Labsphere USS-600 Uniform Source System incorporating a calibrated lamp and a motorized variable attenuator. ${ }^{9}$ The OLED current-voltage characteristics were measured in a vacuumed metal box.

Figure 2 illustrates a typical OLED dc current-voltage $(I-V)$ characteristic and electroluminescence spectrum (inset). The electroluminescence spectrum clearly shows a peak located at $\sim 570 \mathrm{~nm}$, with a shoulder peak at $\sim 545 \mathrm{~nm}$; the full width at half maximum (FWHM) of this peak is about 80 $\mathrm{nm}$. The $I-V$ curve displays a diode-like behavior, with an ON/OFF current ratio of about $6.7 \times 10^{4}$ at $\pm 25 \mathrm{~V}$. Through the $I-V$ characteristic modeling, we have established that this characteristic cannot be simply described by either one of the following models previously suggested in the literature: space charge limited current (SCLC); trapped charge limited current (TCL); thermionic emission combined with the diode series resistance $R_{s}$; or simple Fowler-Nordheim tunneling. This result implies that none of the above mechanisms is solely dominant in our OLED.

We found that the forward bias current-voltage characteristic can be simply described by the following equations based on the equivalent circuit shown in Fig. 1:

$$
V_{A}=V^{\prime}+V^{\prime \prime} \text { with } R_{S} \approx 0
$$

\footnotetext{
a) Applied Physics Program.

b)Electronic mail: Kanicki@eecs.umich.edu
} 


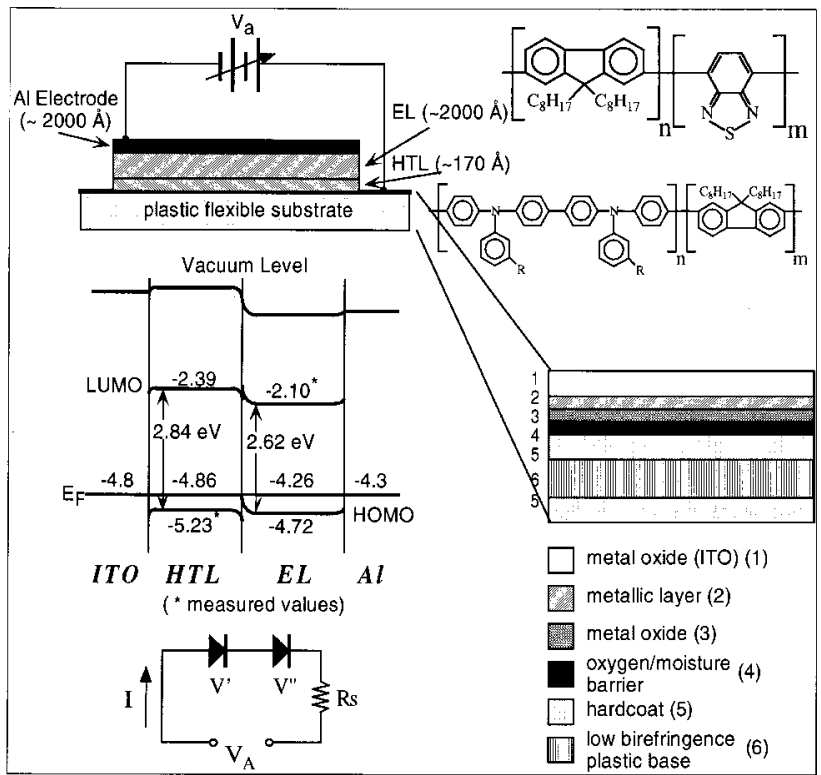

FIG. 1. The OLED bilayer structure, the polymers chemical structures, the schematic energy band diagram of the OLED in equilibrium, the crosssection of the plastic substrate, and the equivalent circuit of the OLED are shown. The EL and HTL polymers are benzothiadiazole-fluorene and amine-fluorene copolymer, respectively. The "*”, represents measured values.

$$
\text { and } V^{\prime}=V_{0}^{\prime} \ln \left(\frac{I}{I_{0}^{\prime}}+1\right), \quad V^{\prime \prime}=V_{0}^{\prime \prime} \ln \left(\frac{I}{I_{0}^{\prime \prime}}+1\right) \text {, }
$$

where $V_{0}^{\prime}\left(=S^{\prime} k T / q\right)$ and $V_{0}^{\prime \prime}\left(=S^{\prime \prime} k T / q\right)$ are the fitting parameters; $S^{\prime}$ and $S^{\prime \prime}$ are the slopes of the $I-V$ characteristics; $k$ is Boltzmann constant, $T(=300 \mathrm{~K})$ is the temperature, $q$ is the charge of electron, and $I_{0}^{\prime}$ and $I_{0}^{\prime \prime}$ are constants. The $V^{\prime}$ and $V^{\prime \prime}$ represent the voltage drops across the two junctions connected in series, and $R_{s}$ is the equivalent device series resistance. The best fit (solid line in Fig. 2) to the experimental data has been obtained for the following fitting parameters: $I_{0}^{\prime} \approx 4.07 \times 10^{-9} \mathrm{~mA} / \mathrm{cm}^{2}, \quad I_{0}^{\prime \prime} \approx 2.27 \times 10^{-2} \mathrm{~mA} / \mathrm{cm}^{2}$, $V_{0}^{\prime} \approx 0.44$, and $V_{0}^{\prime \prime} \approx 3.35$.

The above equations describe the deep gap state (defects)-assisted multistep tunneling process ${ }^{10}$ that can take place across both junctions; and the $V_{0}^{\prime}$ and $V_{0}^{\prime \prime}$ parameters represent the number of steps (involving a series of closely spaced deep gap defects) that carrier must travel through a

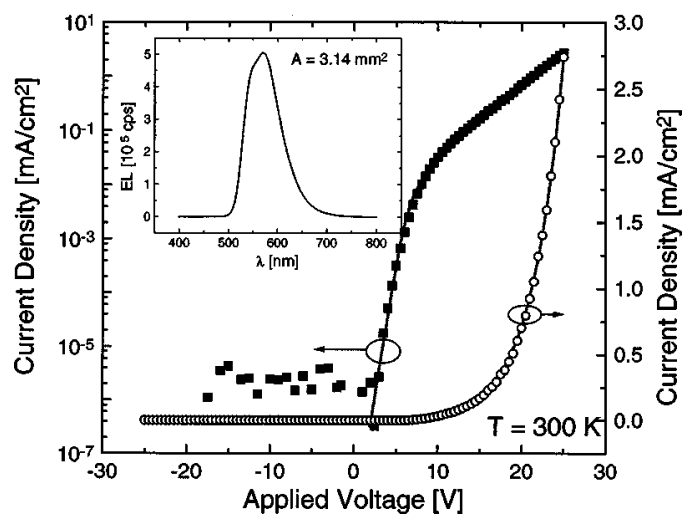

FIG. 2. The experimental $(\boldsymbol{\square}, \bigcirc)$ and simulated $(-)$ current-voltage characteristics of the bilayer OLED are given. The inset shows the electroluminescence spectrum of the OLED collected by the CCD system. given junction. These defects can be associated with the impurities and/or carbon or nitrogen point defects. In our case we assume that the HTL/EL junction is behaving like a $p^{+}-p^{-}$heterojunction, while the EL/Al junction is acting as a typical metal-semiconductor junction. Also the ITO/HTL $\left(p^{+}\right.$-like) junction is assumed to be ohmic-like in our OLEDs, and therefore this contact will not provide any significant contribution to the $I-V$ characteristic. Hence, at a constant electrical field the OLED carrier current is mainly limited by both junctions (e.g., multistep carrier tunneling/ hopping through the HTL/EL junction and electron injection followed by the multistep tunneling/hopping through the EL/Al junction), rather than by the carrier transport through the polymer bulk; although the influence of the deep gap states-assisted bulk carrier transport can also be important in the OLED. In agreement with our experimental data the OLED injected current level (and the OLED brightness) is indeed higher if the low work function metals are used. ${ }^{7}$

In the forward bias conditions, i.e., ITO is positively biased with respect to $\mathrm{Al}$, the light emission occurred when the applied voltage is greater than $\sim 6 \mathrm{~V}$. No light emission is observed when the reversed bias is applied to the OLED structure, i.e., Al is positively biased with respect to ITO. In this case, the reverse resistance of the OLED is large. According to the Ohm's law, a reverse voltage divided by a large device resistance yields a very small reverse current (e.g., $4.1 \times 10^{-4} \mathrm{~mA} / \mathrm{cm}^{2}$ at $-25 \mathrm{~V}$ in Fig. 2) that is insufficient to cause the light emission. A certain current density level (around $0.01 \mathrm{~mA} / \mathrm{cm}^{2}$ in our case) is needed to produce the visible light. The electrons and holes that are injected, and transported through the junctions by multisteps process under the forward bias, into the polymer LUMO and HOMO bands are relaxed to negatively and positively charged polarons through electron-phonon lattice coupling. These polarons will move toward each other under the influence of the applied electrical field and they will recombine on a certain segment of polymer chain to form the singlet excitons. Through the resonance interactions, these singlet excitons can form the excimers, which will emit light Stokes shifted with respect to the absorption spectrum. ${ }^{11}$ The proposed mechanism of the light generation is in agreement with our experimental data. 6,7

Figure 3 shows a typical variation of the OLED brightness (or luminance, $L$ in $\mathrm{cd} / \mathrm{m}^{2}$ ) with the applied current density (the inset shows the OLED spectral distribution of luminance obtained at the different applied current densities). The integration of the OLED spectral distribution of luminance over the wavelength yields the luminance of the OLED. A near-linear relationship obtained between the luminance and the applied current density $\left(J\right.$ in $\left.\mathrm{mA} / \mathrm{cm}^{2}\right)$, with a gradual saturation at $J \geqslant 5 \mathrm{~mA} / \mathrm{cm}^{2}$, can be described by the following equation:

$$
L \cong A J+B J^{2},
$$

where $A(354 \pm 28)$ and $B(-14 \pm 3)$ are constants. At the low current injection this equation can be approximated by: $L \propto J$; and at the high current injection the light-emission saturates at a certain level (in our case at $\sim 2300 \mathrm{~cd} / \mathrm{m}^{2}$ for 


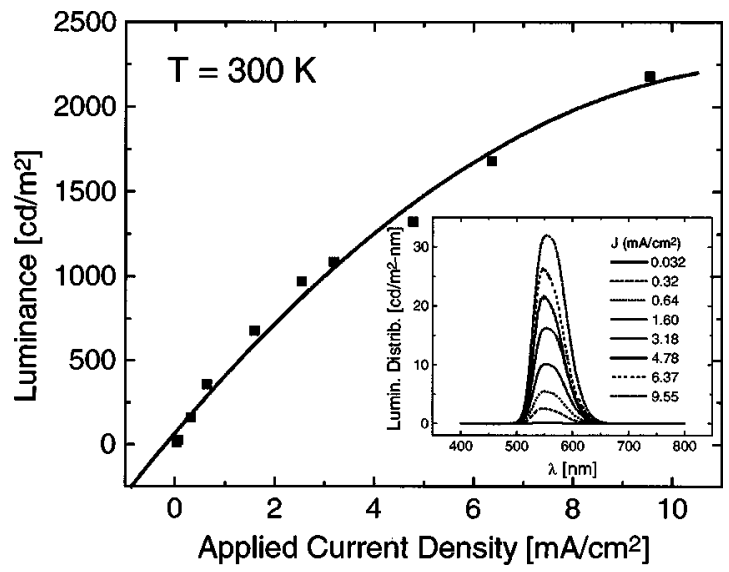

FIG. 3. The evolution of the OLED brightness as a function of the operating current density is shown. The solid line represents the fit of the experimental data to Eq. (3). The inset shows the OLED luminance distribution spectra for different applied current densities.

$J \approx \sim 12.6 \mathrm{~mA} / \mathrm{cm}^{2}$ ). The saturation in this curve is due to the OLED heating that is usually observed at the high current injection levels. ${ }^{12}$

The evolution of the OLED external quantum efficiency ( = number of the photons emitted/electron supplied by the external circuit, e.g., applied current density) at different applied current densities is shown in Fig. 4 (the inset illustrates the photon density distribution spectra at the different applied current densities). The integration of the areas under the photon density distribution provides the number of photons emitted per unit area. We can conclude from this figure that the OLED emission efficiency increases rapidly at first then drops down gradually and starts slowly to flat out at about $4.78 \mathrm{~mA} / \mathrm{cm}^{2}(27.7 \mathrm{~cd} / \mathrm{A})$ with the increasing applied

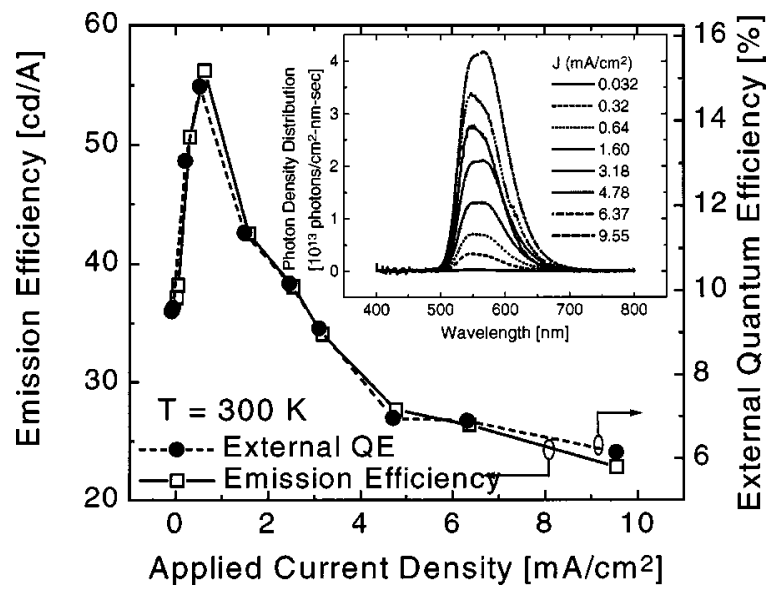

FIG. 4. The evolution of the OLED emission efficiency ( $\square$ ) and external quantum efficiency $(\bullet)$ as a function of the applied current density are shown. The inset shows the OLED photon density distribution spectra for different applied current densities. current density (or luminance). The evolution of device external quantum efficiency also follows the same trend with the applied current density, and the maximum emission efficiency, luminous, and external quantum efficiencies for our OLED are $\sim 56.2 \mathrm{~cd} / \mathrm{A}, \sim 8.96 \mathrm{~lm} / \mathrm{W}$, and $\sim 14.8 \%$, respectively. This excellent OLED performance could be obtained through the development of the following technologies for this device: minimum ITO surface roughness [root-meansquare (rms) $\sim 2.85 \mathrm{~nm}$ measured over $\left.\sim 30 \times 30 \mu \mathrm{m}^{2}\right]$; thin flexible substrate $(\sim 0.2 \mathrm{~mm})$; adequate refraction index matching between polymer $(\sim 1.72)$ and substrate; good control of the polymer morphology (aggregated species) during spin coating through the optimization of the solvent concentration of the polymer solution and the spin speed; and adequate electron transport within the EL polymer through the introduction of benzothiadiazole group (electronwithdrawing group), e.g., EL polymer is used for both the electron transport and light emission. The reduction of the external quantum efficiency at the higher current density is due to the light-induced defects creation (bond breaking) and/or device internal field modification leading to reduction of the density of the excimer states.

We would like to thank Dr. R. Hattori and Dr. S. Gong for their technical assistance during this project. All devices have been fabricated at the University of Michigan Electronics Manufacturing Laboratory. The organic polymers used in this study have been provided by the Dow Chemical Company (Midland, MI). The plastic film conductor was provided by Polaroid Corporation. This research project was financially supported by the Applied Physics Program and the Center for Display Technology and Manufacturing at the University of Michigan, Ann Arbor, and DARPA-ONR grant (N0014-99-1-0958).

${ }^{1}$ F. Matsumoto, T. Nagata, T. Miyabori, H. Tanaka, and S. Tsushima, SID 93 Digest, 1993, p. 965.

${ }^{2}$ N. D. Young, R. M. Bunn, R. W. Wilks, D. J. McCulloch, S. C. Deane, M. J. Edwards, G. Harkin, A. D. Pearson, J. Soc. Inf. Disp. 5/3, 275 (1997).

${ }^{3}$ G. Gustafsson, Y. Cao, G. M. Treacy, F. Klavetter, N. Colaneri, and A. J. Heeger, Nature (London) 357, 477 (1992).

${ }^{4}$ G. Gustafsson, G. M. Treacy, Y. Cao, F. Klavetter, N. Colaneri, and A. J. Heeger, Synth. Met. 55-57, 4123 (1993).

${ }^{5}$ A. Berntsen, Y. Croonen, C. Liedenbaum, H. Schoo, R-J. Visser, J. Vleggaar, and P. Weijer, Opt. Mater. 9, 125 (1998).

${ }^{6}$ Y. He, S. Gong, R. Hattori, and J. Kanicki, Appl. Phys. Lett. 74, 2265 (1999).

${ }^{7}$ Y. He and J. Kanicki, Proceedings of Euro Display'99, 1999, p. 143.

${ }^{8}$ P. Y. Z. Chu, H. C. Choi, L. S. Health, C. S. Ko, J. Mack, P. Nagarkar, J. Richard, W. Smyth, and T. Wang, SID 98 Digest, 1998, p. 1099.

${ }^{9}$ Y. He, R. Hattori, and J. Kanicki (unpublished).

${ }^{10}$ J. Kanicki, Handbook of Conducting Polymers, edited by T. A. Skotheim (Dekker, New York, 1986), p. 543.

${ }^{11}$ Practical Fluorescence, edited by G. G. Guilbault (Dekker, New York, 1990).

${ }^{12}$ P. W. M. Blom and M. J. M. De Jong, Philips J. Res. 51, 479 (1998). 